Non-Invasive Brain Stimulation Targeting the Right Fusiform Gyrus

Selectively Increases Working Memory for Faces

\author{
Tad T. Brunyé ${ }^{1,2,3}$ \\ Joseph M. Moran ${ }^{1,2}$ \\ Amanda Holmes ${ }^{1,2}$ \\ Caroline R. Mahoney ${ }^{1,2}$ \\ Holly A. Taylor ${ }^{1,3}$
}

${ }^{1}$ Center for Applied Brain \& Cognitive Sciences, Tufts University, 200 Boston Ave., Suite 3000, Medford, MA 02155 USA

${ }^{2}$ U.S. Army Natick Soldier Research, Development, and Engineering Center, RDNS-SEW-THC, 15 General Greene Ave, Natick, MA USA

${ }^{3}$ Tufts University, Department of Psychology, 490 Boston Ave., Medford, MA USA

\title{
Contact Author
}

Tad T. Brunyé, Ph.D.

Center for Applied Brain \& Cognitive Sciences

200 Boston Ave., Suite 3000

Medford, MA 02155

tbruny01@,tufts.edu

telephone: 617-306-6262

fax: $617-627-3181$ 


\begin{abstract}
The human extrastriate cortex contains a region critically involved in face detection and memory, the right fusiform gyrus. The present study evaluated whether transcranial direct current stimulation (tDCS) targeting thisanatomical regionwould selectively influence memory for faces versus non-face objects (houses). Anodal tDCS targeted the right fusiform gyrus (Brodmann's Area 37), with the anode at electrode site PO10, and cathode at FP2. Two stimulation conditions were compared in a repeated-measures design: $0.5 \mathrm{~mA}$ versus $1.5 \mathrm{~mA}$ intensity; a separate control group received no stimulation. Participants completed a working memory task for face and house stimuli, varying in memory load from 1 to 4 items. Individual differences measures assessed trait-based differences in facial recognition skills. Results showed $1.5 \mathrm{~mA}$ intensity stimulation (versus $0.5 \mathrm{~mA}$ and control) increasedperformance at high memory loads, but only with faces. Lower overall working memory capacity predicted a positive impact of tDCS.Results provide support for the notion of functional specialization of the right fusiform regions for maintaining face (but not non-face object) stimuli in working memory, and further suggest that low intensity electrical stimulation of this region may enhance demanding face working memory performance particularly in those with relatively poor baseline working memory skills.
\end{abstract}

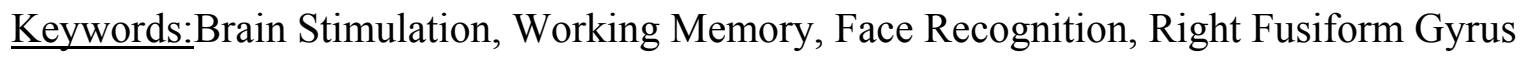


Non-Invasive Brain Stimulation Targeting the Right Fusiform Gyrus

Selectively Increases Working Memory for Faces

\section{Introduction}

The ability to detect and remember faces is critical to human social functioning, allowing us to know when people are present, recognize a familiar face, and identify known individuals (Kanwisher \& Yovel, 2006). These processes involve a network of interrelated brain regions including at least the inferior occipital gyrus, superior temporal sulcus, and lateral fusiform gyrus (Haxby, Hoffman, \& Gobbini, 2002). The most consistently implicatedregion in this network is the right fusiform gyrus, a portion of which has been termed the "fusiform face area" (FFA) by Kanwisher and colleagues (Kanwisher, McDermott, \& Chun, 1997). Though subject to debate(Gauthier, Tarr, Anderson, Skudlarski, \& Gore, 1999; Kanwisher \& Yovel, 2006) this region is thought to serve as a specialized module for face perception, responding maximally to the perception of face stimuli relative to non-face objects such as houses (Haxby et al., 1999), cars (Grill-Spector, Knouf, \& Kanwisher, 2004), orflowers (McCarthy, Puce, Gore, \& Allison, 1997).

One popular method for examining the specialized ability for individuals to process and maintain faces is to engage them in a working memory task with varying load (e.g., maintaining 1-4 faces), and varying stimulus domains (e.g., maintaining faces versus houses)(Druzgal \& D’Esposito, 2001, 2003; Gazzaley, Rissman, \& D’Esposito, 2004; Ranganath, DeGutis, \& D'Esposito, 2004). Overall, research has demonstrated parametrically increasing right fusiform gyrus activity (with fMRI) as working memory set size increases from 1 to 4 faces (Druzgal \& D'Esposito, 2003), and much greater responses in this region to faces than houses (Yovel \& Kanwisher, 2004). Thus, the right fusiform gyrus appears to be involved in the processing and 
maintenance of faces during a working memory task. Transcranial direct current stimulation (tDCS) provides a unique opportunity to explore causal relationships between right fusiform gyrus activity and working memory for faces, by selectively modulating brain activity in this region and measuring the impact the processing and maintenance of faces versus non-face objects.

Transcranial direct current stimulation (tDCS) involves non-invasively applying low intensity electrical current to brain regions by way of electrodes positioned on the surface of the scalp (Brunoni et al., 2012). In most studies, between 1.0-2.0mA intensity direct current (DC) is administered by positioning electrodes in a manner intended to target brain regions of interest with anodal or cathodal polarity stimulation (Jacobson, Koslowsky, \& Lavidor, 2012).A unidirectional flow of charge emanates from a single anode, propagates through cortical tissue, and returns via a single cathode; as anodal currentpropagates through the cortex, it produces neuronal membrane depolarization (Purpura \& McMurtry, 1965), increases neuronal firing rates(Nitsche \& Paulus, 2000, 2001), and increases functional brain connectivity intask-related networks(Peña-Gómez et al., 2012). Though tDCS has been used extensively in the behavioral, cognitive, clinical, and affective sciences literature (Jacobson et al., 2012; Price, McAdams, Grossman, \& Hamilton, 2015; Shiozawa et al., 2014), very few studies have examined its influence on face processing or memory.In two of them, anodal tDCS was applied over the dorsolateral prefrontal cortex (Lafontaine, Theoret, Gosselin, \& Lippe, 2013) or occipitotemporal cortex (which includes the FFA, the occipital face area (OFA), and superior temporal sulcus (Yang et al., 2014)), and outcomes were measured via event-related potentials (ERPs). In the first experiment, the authors found enhanced N170 repetition suppression, indicating faster face recognition with right hemisphere anodal stimulation. In the second experiment, the authors 
found anodal or cathodal stimulation to influence the N170 waveform during a face orientation task, an effect maximal over the right hemisphere. In a more recent study, Renzi and colleagues (Renzi et al., 2015)used anodal OFA stimulation and found reduced performance on a task involving the detection and discrimination of faces and non-face objects; right fusiform gyrus stimulation was not examined (see also (Barbieri, Negrini, Nitsche, \& Rivolta, 2015)). Thus, existing research regarding putative tDCS influences on face processing and memory is equivocal, using varied stimulation targets and outcome measures, and finding mixed results.

Though tDCS has not yet been applied in the context of working memory for face stimuli, or for specifically targeting the right fusiform gyrus, an extensive literature examines the impact of tDCS on working memory task performance. Indeed one of the most reliable results in the tDCS literature is an enhancement of verbal $n$-back task performance with tDCS targeting the left dorsolateral prefrontal cortex (DLPFC) (Berryhill, Peterson, Jones, \& Stephens, 2014; Brunoni \& Vanderhasselt, 2014). For instance, Ohn and colleagues found higher accuracy on a verbal $n$-back task with anodal versus sham tDCS targeting the left DLPFC, and Mulquiney and colleagues found faster response times on a verbal $n$-back task with anodal versus sham tDCS targeting the left DLPFC. This pattern is largely restricted to letter, digit, and word stimuli, though it is generally attributed to this brain region's involvement in implementing attentional control strategies during working memory tasks (Callicott et al., 1999).

The present study was designed to build upon extant studies in four primary ways. First, we attempted to selectively target the right fusiform gyrus using algorithms that predict tDCS current propagation through cortical structures; no other studies have targeted this region in this manner. Though imperfect, these models afford some prediction of maximal focality and intensity at targeted brain structures (Datta et al., 2009; Dmochowski, Datta, Bikson, Su, \&Parra, 
2011). Second, we used outcome measuresthat allow understanding tDCS influences on both face and non-face object working memory. Specifically, we adopted a task involving the delayed recognition of both faces and houses (Yovel \& Kanwisher, 2004) with varied working memory load (i.e., set size). Previous fMRI research shows parametrically increasing right fusiform gyrus activity as face set size increases from 1 to 4 faces (Druzgal \& D’Esposito, 2003), and much greater responses in this region to faces than houses(Yovel \& Kanwisher, 2004). This research suggests that tDCS targeting this region may selectively impact face, but not house working memory, and that any tDCS impacts on face working memory may be load-contingent. If so, such results would provideunique causal evidence for the stimulus-specific and load-contingent role of the right fusiform gyrus during working memory tasks demanding the processing and maintenance of faces.

Third, we used individual differences measures to evaluate whether trait face recognition skills might predict the magnitude of our effects; recent studies suggest that individual differences can be valuable in understanding sometimes unreliable tDCS influences on perception and cognition(Berryhill \& Jones, 2012; Brunyé, 2015; Brunyé, Holmes, et al., 2014; Jones, Gozenman, \& Berryhill, 2015; Slaby et al., 2015).Understanding baseline individual skills on domain-general (e.g., working memory) and domain-specific (e.g., face processing) tasks can provide predictive value for the impact of tDCS. For instance, recent research shows that tDCS of right medial temporal lobe regions only improves navigation performance for individuals with relatively poor spatial sense of direction (Brunyé, Holmes, et al., 2014), and tDCS of DLPFC only improved arithmetic performance for individuals with high mathematics anxiety (Sarkar, Dowker, \& Cohen Kadosh, 2014). It is unknown how individual differences in working memory ability may modulate the influence of tDCS on face working memory task performance.Finally, 
we incorporate a mixed experimental design that controls for perceived cutaneous sensation and reduces the potential for experimental demand characteristics influencing arousal and task performance (Brunyé, Cantelon, Holmes, Taylor, \& Mahoney, 2014).

While performing the working memory task (i.e., online), participants received either $0.5 \mathrm{~mA}$ or $1.5 \mathrm{~mA}$ active anodal tDCS, in a repeated-measures (crossover) design. We chose this design based on a review of recent literature. First, there is compelling evidence that online stimulation influences cognitive task performance either similarly to (Axelrod, Rees, Lavidor, Bar, \& Corballis, 2015; Wirth et al., 2011), or more than (Martin, Liu, Alonzo, Green, \& Loo, 2014), offline tDCS. Second, an anodal-excitatory effect is reliably obtained in cognitive literature (Jacobson et al., 2012), and we chose $1.5 \mathrm{~mA}$ because it reliably influences cortical excitability (Dymond, Coger, \& Serafetinides, 1975)and cognitive task performance (Ditye, Jacobson, Walsh, \& Lavidor, 2012; Javadi, Cheng, \& Walsh, 2012); furthermore, the influence of tDCS does not necessarily scale with increased intensity to 2.0mA (Batsikadze, Moliadze, Paulus, Kuo, \& Nitsche, 2013).Finally, we chose to use a low intensity stimulation control condition $(0.5 \mathrm{~mA})$ rather than sham due to recent research demonstrating cutaneous sensation differences in sham versus active stimulation conditions, and unintentional participant awareness of condition-based differences (Brunyé, Cantelon, et al., 2014). A 0.5mA low intensity control condition reduces otherwise large differences in perceived sensation between active and sham conditions, without influencing cognitive task performance relative to sham (Brunyé, Cantelon, et al., 2014; Nitsche \& Paulus, 2000).In fact, to our knowledge only one sleep-related study suggests an influence of $0.5 \mathrm{~mA}$ stimulation on any cognitive, behavioral, or physiological measures (Marshall, Mölle, Hallschmid, \& Born, 2004). To ensure no discernable influence of 
0.5mA stimulation on task performance, we also collected control data from a separate group of participants who received no stimulation.

We expect that tDCS targeting the right fusiform gyrus will influence performance on the working memory task; given the putative face-selective role of this region, we also expected this effect to be specific to faces, and not extend to houses. This effect may also emerge only at relatively high memory set sizes, given possible ceiling performance at low memory loads (Druzgal \& D’Esposito, 2003),parametric increases in right fusiform gyrus activation with increasing memory loads (Druzgal \& D’Esposito, 2003), and literature demonstrating a more reliable impact of tDCS on relatively difficult working memory tasks (Berryhill et al., 2014).It could also be the case that higher working memory loads with category-specific face stimuli necessitate increased engagement of the fusiform gyrus, whereas category-general working memory stimuli may not. If so, then we might also expect that individuals with relatively poor performance at high face memory loads may stand to benefit most from stimulation. For individual differences, no particular hypotheses were made with regard to tDCS influences, though we did expect that individuals with higher trait face recognition skills (Duchaine \& Nakayama, 2006)would also demonstrate higher performance on the delay-recognition task. It could also be the case that high or low trait face recognition skills would produce ceiling or floor effects on our task, respectively, reducing any particular influence of tDCS.

\section{Materials \&Methods}

\subsection{Participants}

Twenty-four healthy subjects participated (15 female, 9 male, age $21.3+/-3.3)$ in the main experiment for monetary compensation. An additional 24 healthy subjects formed the control 
group to gain baseline data on the working memory task (17 female, 7 male, age $19.6+/-$

3.6).Participants were randomly assigned to the main experimental versus control groups. Written informed consent was provided, in accordancewith approvals by the Tufts University Institutional Review Board and U.S. Army Human Research Protections Office.

\subsection{Experimental design}

Participants were assigned to one of two groups. The control group received no stimulation. The experimental group received anodal tDCS while completing the working memory task.

All participants completed a test to evaluate individual differences in face recognition abilities, and then the working memory task. To evaluate individual differences we administered the Cambridge Face Memory Test (CFMT) (Duchaine \& Nakayama, 2006), which involves viewing a series of faces and then a forced-choice response regarding which of 3 probe faces matches one previously seen. The test reliably assesses face recognition skills across a range of abilities. Following the CFMT, all participants performed a working memory task with a varied set size (memory load) ranging from 1 to 4 faces or houses(Druzgal \& D’Esposito, 2003; Yovel \& Kanwisher, 2004); we included houses (Yovel \& Kanwisher, 2004) to examine the specificity of any tDCS effects. A total of 144 grayscale faces were gathered from the original (Georghiades, Belhumeur, \& Kriegman, 2001) and extended (Lee, Ho, \& Kriegman, 2005) Yale Face Database $\mathrm{B}$, and 144 houses (converted to grayscale) from Google ${ }^{\mathrm{TM}}$ Image searches across multiple architectural categories (split/bilevel, bungalow, colonial, ranch, and farmhouse). All images were cropped to limit contextual features (hair, ears, neighboring homes). As depicted in Figure 1, a trial involved encoding a sequence of 4 stimuli, containing from 1 to 4 faces or houses, then 
responding to a single face or house probe. Filler stimuli to be used in trials containing fewer than 4 faces or houses were created by pixel scrambling (at 24x24 pixels) all 288 images.

The working memory task involved responding to 96 trials: 48 face trials and 48 house trials; trial types were mixed (i.e., not blocked), trial order was random, and no feedback was provided. Each trial displayed a sequence of 4 stimuli (including from 1 to 4 faces or houses) for 1 second each with a $200 \mathrm{~ms}$ ISI. Then, there was a 4 -second delay followed by a memory probe, to which participants responded Yes or No using right and left index fingers on a Logitech F310 gaming controller. If they recognized the probe as presented in the preceding sequence, they were instructed to press Yes, and if not they pressed No. They were instructed to respond as quickly as possible without compromising accuracy. Each trial contained 1, 2, 3, or 4 faces or houses, with $3,2,1$, or 0 filler items, respectively, randomly ordered within the trial. The number of faces or houses in a given trial is the memory load manipulation. Half of the trials used a previously presented "old" probe (to elicit a Yes), and half used a foil "new" probe (to elicit a No). PsychoPy (Peirce, 2007) was used for all image presentation and response logging.

\subsubsection{Procedure}

The control group visited the laboratory for two sessions, separated by at least 24 hours. During the first visit they completed the CFMT and working memory task; during the second visit, they again completed the working memory task. A brief 4-trial practice task with feedback was provided before administering the main working memory task. For the control group, no tDCS was administered during either session.

The experimental group also visited the laboratory for two sessions, separated by at least 24 hours (minimizing the possibility of carry-over effects between sessions (Monte-Silva et al., 
2013)). As done with the control group, during the first visit they completed the CFMT and working memory task, and during the second visit only the working memory task. Practice working memory trials were completed prior to the task. During each session, participants experienced one of the two counterbalanced stimulation conditions $(0.5 \mathrm{~mA}, 1.5 \mathrm{~mA})$. An appropriately sized tDCS cap was fitted to the participant and condition-specific stimulation began; the experimenter manually ramped up to the target stimulation intensity over the course of 1 minute in increments corresponding to $33 \%$ target intensity (i.e., beginning at $0.5 \mathrm{~mA}$, then to $1 \mathrm{~mA}$, then to $1.5 \mathrm{~mA}$ ). After an additional 3 minutes,participants provided a perceived sensation rating on a scale from 0 (Cold) to 9 (Hurts a lot) (Clark et al., 2012), and then began the working memory task.

\subsection{2. $\mathrm{tDCS}$}

To define our electrode montage we targeted the right fusiform gyrus using the HDTargets software package (Soterix Medical, Inc.), optimized for maximum intensity at target. Predicted current propagation is depicted in Figure 2, with the functionally-defined FFA circled. With electrodes positioned at PO10 (anode) and Fp2 (cathode), the model predicted a field intensity of $0.44 \mathrm{~V} / \mathrm{m}$ at target. tDCS was administered using the $\mathrm{M} \times \mathrm{N}$ high-definition tDCS system manufactured by Soterix Medical, Inc. (New York, NY). Two Ag/AgCl sintered ring electrodes were mounted on a 74-channel EasyCap (EEG cap) using plastic holders. Signa gel (Parker Laboratories, Fairfield, NJ) was used to conduct current between the electrode and scalp, and reduce impedances $(<2 \mathrm{k} \Omega)$. 


\subsubsection{Statistical analysis.}

The CFMT is scored as percentage correct out of the 72 trials. For the working memory task, we calculated Cowan's $K$ as a measure of the number of items held in memory(Cowan, 2000), and response times (RTs) for correctly answered trials. Note that sensitivity-related measures (dprime, response criterion) are also reported in Table 1. Cowan's K and RT data were separately entered intotwo repeated-measures analyses of variance (ANOVAs) withStimulus Category (2: Faces, Houses), Stimulation Condition (2: $0.5 \mathrm{~mA}, 1.5 \mathrm{~mA})$, and Memory Load (1,2,3,4 stimuli) as repeated measures. To assess whether individual differences in trait face recognition skills predict performance on the working memory task and the influence of stimulation, we entered CFMTscores as predictors into three regressions. The first and second examined whether CFMT scores predicted Cowan's $K$ and RTs. The third examined whether CFMT scores predicted the effect size (Cohen's $d$ ) comparing Stimulation Conditions (1.5mA versus $0.5 \mathrm{~mA})$ at the level of individual participants. All statistical results are accompanied by effect sizes in the form of etasquared $\left(\eta^{2}\right)$ or Cohen's $d$. When considering control group data, note that to account for any learning effects we have collapsed across this group's two sessions.

\section{Results}

Table 1 details mean and standard deviation Cowan's $K$,hit rates, false alarm rates, sensitivity (dprime), response criterion, and response times, for all conditions and groups.

\subsection{Perceived Sensation.}

In the experimental group, perceived sensation did not differ as a function of Session, $F(1,23)=$ $.14, p=.72, \eta^{2}<.01$, or Stimulation Condition, $F(1,23)=.82, p=.38, \eta^{2}=.03$. Critically, participants did not perceive cutaneous sensation differences between the $0.5 \mathrm{~mA}(M=2.88, S D$ 
$=1.2)$ and $1.5 \mathrm{~mA}(M=3.23, S D=1.6)$ stimulation intensities, reducing the likelihood of unintentional task demands influencing participant performance. In the control group, we did not collect perceived sensation ratings.

\subsection{CFMT.}

Mean scores did not differ between the control $(M=58.8, S D=8.8)$ and experimental $(M=59.9$, $S D=8.2)$ groups, $t(46)=.47, p=.64, d=.14$.

\subsection{Experimental Group: Working Memory Task.}

For Cowan's $K$, there were main effects of Stimulation, $F(1,23)=6.79, p=.016, \eta^{2}=.01$, and Memory Load, $F(3,69)=92.87, p<.001, \eta^{2}=.49$. These effects were qualified by two2-way interactions, one interaction between Stimulus Category and Stimulation, $F(1,23)=6.98, p=$ $.015, \eta^{2}<.01$, and another interaction between Stimulus Category and Memory Load, $F(3,69)=$ $3.33, p=.024, \eta^{2}=.01$.

To examine the first interaction between Stimulus Category and Stimulation, we conducted two simple effects ANOVAs with Stimulation Condition as the single factor, one within the faces Stimulus Category, and the other within the houses Stimulus Category. For faces, there was an effect of Stimulation Condition, $F(1,23)=20.59, p<.001, \eta^{2}=.47$, as depicted in Figure 3.To explore the pattern in Figure 3 and specifically test our hypotheses, we used paired t-testswith a conservative Bonferroni correction term $(\alpha=.0125)$ to compare the two Stimulation Conditions at each of the four Memory Load levels. Across memory loads, there was increasing evidence for enhanced face memory with $1.5 \mathrm{~mA}$ versus $0.5 \mathrm{~mA}$, particularly at memory loads 3 and 4 ( $p=$ $.088, .032, .01, .004$, for each memory load, respectively). For houses, the effect of Stimulation Condition was non-significant, $F(1,23)=.43, p=.52, \eta^{2}<.01$, as depicted in Figure 4. 
To examine the second interaction between Stimulus Category and Memory Load, we conducted four simple effects ANOVAs with Stimulus Categoryas the single factor, one within each of the Memory Load conditions. Within load levels 1 and 2, there were higher $K$ rates within the faces versus houses conditions, $F(1,23)=7.14, p=.014, \eta^{2}=.24$, and $F(1,23)=4.82, p=.039, \eta^{2}=$ .17 , respectively. There were no differences between faces and houses conditions within load levels 3 or 4 ( $p$ 's $>.13)$.

For RTs, we conducted the same omnibus ANOVA. There was a main effect of Stimulus Category, $F(1,23)=5.21, p<.05, \eta^{2}=.02$, with faster response times to faces $(M=979.2, S D=$ 292.9) than houses $(M=1044.9, S D=336.3)$. There was also a main effect of Memory Load, $F(3,69)=33.85, p<.001, \eta^{2}=.14$, with slower response times at higher memory loads $(M=$ $861.8,1000.8,1082.2$, and 1103.6, respectively). There were no other main or interactive effects $(p ' s>.26)$.

\subsection{Control Group: Working Memory Task.}

Mean Cowan's $K$ rates for the control group are depicted alongside the experimental group means in Figure 3. There was a main effect of Memory Load, $F(3,69)=70.31, p<.001, \eta^{2}=$ .38. All other main and interactive effects were non-significant ( $p$ 's $>.14$ ).

For RTs, there was only a main effect of Memory Load, $F(3,69)=19.2, p<.001, \eta^{2}=.22$, with slower response times at higher memory loads $(M=855.9,966.9,1074.9$, and 1081.3, respectively) (all other $p$ 's $>.05$ ). 


\subsection{Predicting Working Memory Task Performance.}

We first tested whether performance on face trials at the highest working memory load and at $0.5 \mathrm{~mA}$ stimulation intensity predicted the extent of performance improvement with $1.5 \mathrm{~mA}$. In other words, as hypothesized, participants who show relatively low performance at the highest memory load might benefit more from right fusiform gyrus stimulation. To test this hypothesis, we conducted a simple linear regression with $K$ (at $0.5 \mathrm{~mA}$, face trials) as the predictor, and the magnitude of $K$ change between the two stimulation conditions $(1.5 \mathrm{~mA}-0.5 \mathrm{~mA})$ as the dependent variable. The linear regression was significant, with negative predictive value, $F(23)=$ 9.02, $p=.007, R=.54, \beta_{\text {std }}=-.54$. Thus, lower baseline performance in the 4-load condition $(0.5 \mathrm{~mA}$, face trials) predicted greater memory gains with $1.5 \mathrm{~mA}$ stimulation.

Second, we tested whether CFMT scores predicted overall performance on the working memory task with two regressions, one predicting $K$ scores on face trials, and another predicting $K$ scores on house trials. For this test, we combined groups (experimental, control) and stimulation conditions $(0.5 \mathrm{~mA}, 1.5 \mathrm{~mA})$. Though there was some limited suggestion of a positive trend toward higher CFMT scores predicting higher $K$ scores (see Figure 5), this relationship did not reach significance for faces, $F(1,47)=2.56, p=.12, R=.23, \beta_{\text {std }}=.23$, or houses, $F(1,47)=$ 2.07, $p=.16, R=.21, \beta_{\text {std }}=.21$.

Finally, we tested whether CFMT scores predicted the influence of tDCS on face or house trials in the experimental group. Our predictor wasCFMT scores, and our dependent variable was the difference between $K$ rates in the $1.5 \mathrm{~mA}$ versus $0.5 \mathrm{~mA}$ stimulation conditions (i.e., $1.5 \mathrm{~mA}$ minus $0.5 \mathrm{~mA}$ for each participant). In this manner, higher difference scores indicate a larger influence of tDCS on face memory. For faces, the regression model was non-significant, $F(1,23)=.98, p$ 
$=.33, R=.21, \beta_{\text {std }}=.21$; the same non-significance was found for houses, $F(1,23)=.14, p=.71$, $R=.08, \beta_{\text {std }}=.08$.

\subsection{Comparison to Control Group}

We tested whether $1.5 \mathrm{~mA}$ stimulation increased $K$ rates for face trials relative to the control group, at each memory load. A series of four independent samples t-tests (using a Bonferroni correction term, $\alpha=.0125$ ) demonstrated higher $K$ rates in the $1.5 \mathrm{~mA}$ condition relative to the control group at memory load $4, \mathrm{t}(46)=3.05, p=.004, d=.88$. The other three comparisons did not reach significance ( $p$ 's $=.21, .04, .03$ for loads 1,2 , and 3 , respectively). Comparing the $0.5 \mathrm{~mA}$ condition to the control group was non-significant at all memory loads ( $p$ 's $>.56$ ).

We conducted these same tests with data from house trials. Overall $K$ rates did not differ between the $1.5 \mathrm{~mA}$ condition and control group at any memory load (all $p$ 's $>.21$ ), or between the $0.5 \mathrm{~mA}$ condition and control group at any level (all $p$ 's $>.11$ ).

\section{Discussion}

The present study examined whether low current electrical stimulation targeting the right fusiform gyrus would differentially influence performance on a variable load working memory task using face- versus house-based stimuli. We specifically compared $1.5 \mathrm{~mA}$ stimulation to a $0.5 \mathrm{~mA}$ stimulation condition (within-participants), and to a no stimulation control group (between-participants). Both within- and between-participants comparisons demonstrated that $1.5 \mathrm{~mA}$ stimulation increases the number of items stored in memory (using Cowan's K)with the face stimulus type, but not the house stimulus type.This effect was maximal at high memory loads when participants were asked to remember 3 or 4 faces at a time; at load level 4, on average there was an increase of nearly one (.59) additional item maintained in memory with 
$1.5 \mathrm{~mA}$ stimulation. We also examined whether scores on a standardized face recognition task (the CFMT) might predict face memory performance, and predict the influence of tDCS. We only found a non-significant trend suggesting the former, with higher standardized face recognition task scores positively relating to memory performance on face trials. Finally, we also found evidence that participants with relatively low retention at the highest working memory load tended to benefit most from the active $1.5 \mathrm{~mA}$ stimulation condition.

\subsection{Theoretical \& Clinical Implications}

The present results provide support for theoretical positions regarding the role of the right fusiform gyrus in face memory. The notion of specialized brain regions for face processing is controversial (Gauthier et al., 1999; Kanwisher \& Yovel, 2006), with proponents suggestinga functional region of the right fusiform gyrus (the FFA) serving as a specialized module for face perception, responding maximally to face relative to non-face stimuli (Grill-Spector et al., 2004; McCarthy et al., 1997). The present results provide some causal support for this notion, demonstrating that low current electrical stimulation of this region enhances performance on a working memory task specific to faces, and not houses. Had our data demonstrated either increased working memory performance with both stimulus types, or no impact on either stimulus type, we could not confidently lend support for this theory. Of course, this is only weak evidence for functional specificity of this brain region, and we have no evidence that this region is more involved in face recognition than any other particular brain region. We discuss this points more in the Limitations section.

Our overall finding also converges with recent tDCS research stimulating the occipitotemporal cortex (i.e., FFA and OFA) and modulating ERP components related to inverted and upright face processing(Yang et al., 2014). We also found some evidence that the effect of tDCS 
is primarily found at higher memory loads. This particular result supports earlier fMRI research showing parametrically increasing right fusiform gyrus activity as the face memory set size increases from 1 to 4 faces (Druzgal \& D'Esposito, 2003). This pattern also supports emerging consensus in the tDCS literature that the influence of low intensity brain stimulation on working memory tasks is largely contingent on task difficulty (Berryhill et al., 2014). Specifically, low load conditions such as a 0 - or 1-back working memory trials are easily solved and tDCS does not reliably alter performance; however, as working memory task demands increase, the increased neuronal excitability due to anodal tDCS is more likely to impart performance advantages in terms of increased retention and/or decreased response latencies. Of course, this consensus is emerging largely from research using tDCS to target the left DLPFC, and our data provide a unique extension of this finding to a novel stimulation montage and task (for a review, seeBrunoni \& Vanderhasselt, 2014).

Interestingly, we also found that the positive impact of tDCS during the most demanding working memory condition was particularly pronounced when baseline $(0.5 \mathrm{~mA})$ performance in this condition was relatively low. Specifically, participants with lower baseline performance in the 4-face task tended to show more pronounced performance enhancement with $1.5 \mathrm{~mA}$ stimulation.This pattern does not appear to be due to ceiling performance among particular participants; indeed at baseline the vast majority of participants (23/24) showed Cowan's $K$ values less than 3.5 in the 4 -face condition, leaving room for improvement due to stimulation.Rather, our result appears to support an emerging body of evidence that individual differences in task-relevant skills can modulate the influence of tDCS(Brunyé, Holmes, et al., 2014; Sarkar et al., 2014). Thus, tDCS may not produce uniform benefits across individuals with varying baseline skills on a task of interest; continuing research should consider the reliability of 
trait- and skill-based predictors with larger sample sizes and more comprehensive sets of individual differences measures.

The clinical relevance of the present findings is uncertain. While some research demonstrates abnormal right fusiform activation in acquired prosopagnosia during face viewing (Schiltz et al., 2006), still other studies demonstrate normal activation and adaptation in developmental prosopagnosia (Avidan, Hasson, Malach, \& Behrmann, 2005; Hasson, Avidan, Deouell, Bentin, \& Malach, 2003). While the present results showed predictive value of individual differences in face recognition skills (i.e., CFMT scores) on working memory for faces, there was no evidence that variability in face recognition skills predicted the influence of tDCS. Thus, it is unclear whether tDCS would prove a promising therapeutic intervention for individuals with poor face recognition skills. This could be due to the restricted range of CFMT in the present sample; indeed only one of our experimental group participants scored outside of the normal range of CFMT scores (i.e., 43-71; (Duchaine \& Nakayama, 2006)).This participant $($ CFMT $=37)$ showed particularly low performance on the face version of the working memory task $(\mathrm{d}$-prime $=1.47)$, and similar performance across stimulation conditions. Continuing research would benefit from a participant sample with a more diverse range of face recognition skills.

\subsection{Limitations}

There are a few limitations worth considering. First, though current propagation models predict that our electrode montage would result in maximum intensity electrical fields in the right fusiform gyrus, we have no direct evidence that this procedure was successful in targeting this region across participants. For instance, the present results might be driven by right hemisphere dominance in face processing (Behrmann \& Plaut, 2014; Rossion, Joyce, Cottrell, \& Tarr, 
2003)relative to other object stimuli not demanding expert-level categorical or holistic processing (Farah, 2000). However, we do note that recent literature provides compelling evidence validating the specificity of current propagation models (Datta et al., 2009; Dmochowski et al., 2011; Edwards et al., 2013), and our working memory task data provide indirect evidence to this effect. Second, while the present results suggest a selective effect of tDCS on face memory, there are many non-face object categories other than houses that may rely upon the right fusiform gyrus (and other distributed brain regions) for processing (Haxby et al., 2002). For instance exemplars from other expertise-related object categories such as cars, animals, or tools (Bukach, Gauthier, \& Tarr, 2006; Gauthier et al., 1999); the present results cannot speak to whether tDCS targeting the right fusiform gyrus would influence memory performance with such stimuli, and thus cannot speak directly to the domain-general versus domain-specific nature of object processing within this or adjacent brain regions.

\subsection{Conclusions}

We provide the first evidence that anodal tDCS targeting the right fusiform gyrus can improve some aspects of face memory, namely the number of items retained at high memory loads. This pattern was not found with non-face stimuli, and was not predicted by individual differences in face recognition skills. The effect of anodal tDCS, however, was most pronounced in individuals with relatively low working memory capacity for faces. In sum, tDCS targeting the right fusiform gyrus can increase working memory for faces, though continuing research is needed to ascertain the clinical implications of such findings. 


\section{Author Contributions}

T.T.B. and J.M.M. planned and designed the experiment. A.H. recruited and ran participants, and processed data. T.T.B. prepared the manuscript, and J.M.M., C.R.M., and H.A.T. provided critical manuscript revisions.

\section{Acknowledgements}

This work was supported by a grant awarded to H.A.T. from the U.S. Army Natick Soldier Research, Development, and Engineering Center (W911QY-13-C-0012). Permission was granted by the U.S. Army to publish this material. The views expressed in this article are solely those of the authors and do not reflect the official policies or positions of the Department of the Army, the Department of Defense, or any other department or agency of the U.S. government. 


\section{References}

Avidan, G., Hasson, U., Malach, R., \& Behrmann, M. (2005). Detailed exploration of facerelated processing in congenital prosopagnosia: 2. Functional neuroimaging findings. Journal of Cognitive Neuroscience, 17(7), 1150-67. doi:10.1162/0898929054475145

Axelrod, V., Rees, G., Lavidor, M., Bar, M., \& Corballis, M. C. (2015). Increasing propensity to mind-wander with transcranial direct current stimulation. Proceedings of the National Academy of Sciences, 112(11), 3314-3319. doi:10.1073/pnas.1421435112

Barbieri, M., Negrini, M., Nitsche, M. A., \& Rivolta, D. (2015). Anodal-tDCS over the human right occipital cortex enhances the perception and memory of both faces and objects. Neuropsychologia, 81, 238-244. doi:10.1016/j.neuropsychologia.2015.12.030

Batsikadze, G., Moliadze, V., Paulus, W., Kuo, M.-F., \& Nitsche, M. A. (2013). Partially nonlinear stimulation intensity-dependent effects of direct current stimulation on motor cortex excitability in humans. The Journal of Physiology, 591(7), 1987-2000. doi:10.1113/jphysiol.2012.249730

Behrmann, M., \& Plaut, D. C. (2014). Bilateral hemispheric processing of words and faces: Evidence from word impairments in prosopagnosia and face impairments in pure alexia. Cerebral Cortex, 24(4), 1102-1118. doi:10.1093/cercor/bhs390

Berryhill, M. E., \& Jones, K. T. (2012). tDCS selectively improves working memory in older adults with more education. Neuroscience Letters, 521, 148-151.

Berryhill, M. E., Peterson, D. J., Jones, K. T., \& Stephens, J. A. (2014). Hits and misses: leveraging tDCS to advance cognitive research. Frontiers in Psychology, 5, 800. doi:10.3389/fpsyg.2014.00800 
Brunoni, A. R., Nitsche, M. A., Bolognini, N., Bikson, M., Wagner, T., Merabet, L., ... Fregni, F. (2012). Clinical research with transcranial direct current stimulation (tDCS): challenges and future directions. Brain Stimulation, 5(3), 175-95. doi:10.1016/j.brs.2011.03.002

Brunoni, A. R., \& Vanderhasselt, M.-A. (2014). Working memory improvement with noninvasive brain stimulation of the dorsolateral prefrontal cortex: a systematic review and meta-analysis. Brain and Cognition, 86, 1-9. doi:10.1016/j.bandc.2014.01.008

Brunyé, T. T. (2015). Increasing breadth of semantic associations with left frontopolar direct current brain stimulation: a role for individual differences. Neuroreport, 26(5), 296-301.

Brunyé, T. T., Cantelon, J., Holmes, A., Taylor, H. A., \& Mahoney, C. R. (2014). Mitigating cutaneous sensation differences during tDCS: Comparing sham versus low intensity control conditions. Brain Stimulation, 7(6), 832-835. doi:10.1016/j.brs.2014.09.015

Brunyé, T. T., Holmes, A., Cantelon, J., Eddy, M. D., Gardony, A. L., Mahoney, C. R., \& Taylor, H. A. (2014). Direct current brain stimulation enhances navigation efficiency in individuals with low sense of direction. Neuroreport, 25, 1175-1179.

Bukach, C. M., Gauthier, I., \& Tarr, M. J. (2006). Beyond faces and modularity: the power of an expertise framework. Trends in Cognitive Sciences, 10(4), 159-66. doi:10.1016/j.tics.2006.02.004

Callicott, J. H., Mattay, V. S., Bertolino, A., Finn, K., Coppola, R., Frank, J. A., ... Weinberger, D. R. (1999). Physiological characteristics of capacity constraints in working memory as revealed by functional MRI. Cerebral Cortex (New York, N.Y. : 1991), 9(1), 20-6. doi:10.1093/CERCOR/9.1.20

Clark, V. P., Coffman, B. A., Mayer, A. R., Weisend, M. P., Lane, T. D. R., Calhoun, V. D., ... 
Wassermann, E. M. (2012). TDCS guided using fMRI significantly accelerates learning to identify concealed objects. NeuroImage, 59(1), 117-28.

doi:10.1016/j.neuroimage.2010.11.036

Cowan, N. (2000). The magical number 4 in short-term memory: A reconsideration of mental storage capacity. Behavioral and Brain Sciences, 24(1), 87-185. doi:10.1017/S0140525X01003922

Datta, A., Bansal, V., Diaz, J., Patel, J., Reato, D., \& Bikson, M. (2009). Gyri-precise head model of transcranial direct current stimulation: Improved spatial focality using a ring electrode versus conventional rectangular pad. Brain Stimulation, 2(4), 201-207.e1. Retrieved from http://www.sciencedirect.com/science/article/pii/S1935861X09000333

Ditye, T., Jacobson, L., Walsh, V., \& Lavidor, M. (2012). Modulating behavioral inhibition by tDCS combined with cognitive training. Experimental Brain Research, 219(3), 363-8. doi:10.1007/s00221-012-3098-4

Dmochowski, J., Datta, A., Bikson, M., Su, Y., \& Parra, L. (2011). Optimized multi-electrode stimulation increases focality and intensity at target. Journal of Neural Engineering, 8, 046011.

Druzgal, T. J., \& D’Esposito, M. (2001). Activity in fusiform face area modulated as a function of working memory load. Cognitive Brain Research, 10(3), 355-364. doi:10.1016/S09266410(00)00056-2

Druzgal, T. J., \& D’Esposito, M. (2003). Dissecting contributions of prefrontal cortex and fusiform face area to face working memory. Journal of Cognitive Neuroscience, 15(6), 771-784. doi:10.1162/089892903322370708 
Duchaine, B., \& Nakayama, K. (2006). The Cambridge Face Memory Test: results for neurologically intact individuals and an investigation of its validity using inverted face stimuli and prosopagnosic participants. Neuropsychologia, 44(4), 576-85. doi:10.1016/j.neuropsychologia.2005.07.001

Dymond, A. M., Coger, R. W., \& Serafetinides, E. A. (1975). Intracerebral current levels in man during electrosleep therapy. Biological Psychiatry, 10(1), 101-4. Retrieved from http://www.ncbi.nlm.nih.gov/pubmed/1120172

Edwards, D., Cortes, M., Datta, A., Minhas, P., Wassermann, E. M., \& Bikson, M. (2013). Physiological and modeling evidence for focal transcranial electrical brain stimulation in humans: a basis for high-definition tDCS. NeuroImage, 74, 266-75. doi:10.1016/j.neuroimage.2013.01.042

Farah, M. J. (2000). The Cognitive Neuroscience of Vision. Hoboken, NJ: Blackwell.

Gauthier, I., Tarr, M. J., Anderson, A. W., Skudlarski, P., \& Gore, J. C. (1999). Activation of the middle fusiform "face area" increases with expertise in recognizing novel objects. Nature Neuroscience, 2(6), 568-73. doi:10.1038/9224

Gazzaley, A., Rissman, J., \& D’Esposito, M. (2004). Functional connectivity during working memory maintenance. Cognitive, Affective, \& Behavioral Neuroscience, 4(4), 580-599. doi:10.3758/CABN.4.4.580

Georghiades, A. S., Belhumeur, P. N., \& Kriegman, D. (2001). From few to many: Illumination code models for face recognition under variable lighting and pose. IEEE Transactions on Pattern Analysis and Machine Intelligence, 23, 643-660.

Grill-Spector, K., Knouf, N., \& Kanwisher, N. (2004). The fusiform face area subserves face 
perception, not generic within-category identification. Nature Neuroscience, 7(5), 555-62. doi:10.1038/nn1224

Hasson, U., Avidan, G., Deouell, L. Y., Bentin, S., \& Malach, R. (2003). Face-selective activation in a congenital prosopagnosic subject. Journal of Cognitive Neuroscience, 15(3), 419-31. doi:10.1162/089892903321593135

Haxby, J. V., Hoffman, E. A., \& Gobbini, M. I. (2002). Human neural systems for face recognition and social communication. Biological Psychiatry, 51(1), 59-67. doi:10.1016/S0006-3223(01)01330-0

Haxby, J. V, Ungerleider, L. G., Clark, V. P., Schouten, J. L., Hoffman, E. A., \& Martin, A. (1999). The Effect of Face Inversion on Activity in Human Neural Systems for Face and Object Perception. Neuron, 22(1), 189-199. doi:10.1016/S0896-6273(00)80690-X

Jacobson, L., Koslowsky, M., \& Lavidor, M. (2012). tDCS polarity effects in motor and cognitive domains: a meta-analytical review. Experimental Brain Research., 216(1), 1-10. doi:10.1007/s00221-011-2891-9

Javadi, A. H., Cheng, P., \& Walsh, V. (2012). Short duration transcranial direct current stimulation (tDCS) modulates verbal memory. Brain Stimulation, 5(4), 468-74. doi:10.1016/j.brs.2011.08.003

Jones, K. T., Gozenman, F., \& Berryhill, M. E. (2015). The strategy and motivational influences on the beneficial effect of neurostimulation: A tDCS and fNIRS study. NeuroImage, 105, $238-247$.

Kanwisher, N., McDermott, J., \& Chun, M. M. (1997). The Fusiform Face Area: A Module in Human Extrastriate Cortex Specialized for Face Perception. J. Neurosci., 17(11), 4302- 
4311. Retrieved from http://www.jneurosci.org/content/17/11/4302.short

Kanwisher, N., \& Yovel, G. (2006). The fusiform face area: a cortical region specialized for the perception of faces. Philosophical Transactions of the Royal Society of London. Series B, Biological Sciences, 361(1476), 2109-28. doi:10.1098/rstb.2006.1934

Lafontaine, M. P., Theoret, H., Gosselin, F., \& Lippe, S. (2013). Transcranial direct current stimulation of the dorsolateral prefrontal cortex modulates repetition suppression to unfamiliar faces: An ERP study. PLoS One, 8, e81721.

Lee, K. C., Ho, J., \& Kriegman, D. (2005). Acquiring linear subspaces for face recognition under variable lighting. IEEE Transactions on Pattern Analysis and Machine Intelligence, 27, 684-698.

Marshall, L., Mölle, M., Hallschmid, M., \& Born, J. (2004). Transcranial direct current stimulation during sleep improves declarative memory. The Journal of Neuroscience: The Official Journal of the Society for Neuroscience, 24(44), 9985-92. doi:10.1523/JNEUROSCI.2725-04.2004

Martin, D. M., Liu, R., Alonzo, A., Green, M., \& Loo, C. K. (2014). Use of transcranial direct current stimulation (tDCS) to enhance cognitive training: effect of timing of stimulation. Experimental Brain Research, 232(10), 3345-51. doi:10.1007/s00221-014-4022-x

McCarthy, G., Puce, A., Gore, J. C., \& Allison, T. (1997). Face-specific processing in the human fusiform gyrus. Journal of Cognitive Neuroscience, 9(5), 605-10. doi:10.1162/jocn.1997.9.5.605

Monte-Silva, K., Kuo, M.-F., Hessenthaler, S., Fresnoza, S., Liebetanz, D., Paulus, W., \& Nitsche, M. A. (2013). Induction of late LTP-like plasticity in the human motor cortex by 
repeated non-invasive brain stimulation. Brain Stimulation, 6(3), 424-32.

doi:10.1016/j.brs.2012.04.011

Nitsche, M. A., \& Paulus, W. (2000). Excitability changes induced in the human motor cortex by weak transcranial direct current stimulation. The Journal of Physiology, 527(3), 633-639. doi:10.1111/j.1469-7793.2000.t01-1-00633.x

Nitsche, M. A., \& Paulus, W. (2001). Sustained excitability elevations induced by transcranial DC motor cortex stimulation in humans. Neurology, 57(10), 1899-1901. doi:10.1212/WNL.57.10.1899

Peirce, J. W. (2007). PsychoPy - psychophysics software in Python. Journal of Neuroscience Methods, 162, 8-13.

Peña-Gómez, C., Sala-Lonch, R., Junqué, C., Clemente, I. C., Vidal, D., Bargalló, N., ... Bartrés-Faz, D. (2012). Modulation of large-scale brain networks by transcranial direct current stimulation evidenced by resting-state functional MRI. Brain Stimulation, 5(3), 252-263. doi:10.1016/j.brs.2011.08.006

Price, A. R., McAdams, H., Grossman, M., \& Hamilton, R. H. (2015). A Meta-analysis of Transcranial Direct Current Stimulation Studies Examining the Reliability of Effects on Language Measures. Brain Stimulation, 8(6), 1093-100. doi:10.1016/j.brs.2015.06.013

Purpura, D. P., \& McMurtry, J. G. (1965). Intracellular activities and evoked potential changes during polarization of motor cortex. Journal of Neurophysiology, 28, 166-185.

Ranganath, C., DeGutis, J., \& D’Esposito, M. (2004). Category-specific modulation of inferior temporal activity during working memory encoding and maintenance. Cognitive Brain Research, 20(1), 37-45. doi:10.1016/j.cogbrainres.2003.11.017 
Renzi, C., Ferrari, C., Schiavi, S., Pisoni, A., Papagno, C., Vecchi, T., ... Cattaneo, Z. (2015). The role of the occipital face area in holistic processing involved in face detection and discrimination: A tDCS study. Neuropsychology, 29, 409-416.

Rossion, B., Joyce, C. A., Cottrell, G. W., \& Tarr, M. J. (2003). Early lateralization and orientation tuning for face, word, and object processing in the visual cortex. NeuroImage, 20(3), 1609-1624. doi:10.1016/j.neuroimage.2003.07.010

Sarkar, A., Dowker, A., \& Cohen Kadosh, R. (2014). Cognitive enhancement or cognitive cost: Trait-specific outcomes of brain stimulation in the case of mathematics anxiety. Journal of Neuroscience, 34(50), 16605-16610. doi:10.1523/JNEUROSCI.3129-14.2014

Schiltz, C., Sorger, B., Caldara, R., Ahmed, F., Mayer, E., Goebel, R., \& Rossion, B. (2006). Impaired face discrimination in acquired prosopagnosia is associated with abnormal response to individual faces in the right middle fusiform gyrus. Cerebral Cortex (New York, N.Y. : 1991), 16(4), 574-86. doi:10.1093/cercor/bhj005

Shiozawa, P., Fregni, F., Benseñor, I. M., Lotufo, P. A., Berlim, M. T., Daskalakis, J. Z., ... Brunoni, A. R. (2014). Transcranial direct current stimulation for major depression: an updated systematic review and meta-analysis. The International Journal of Neuropsychopharmacology / Official Scientific Journal of the Collegium Internationale Neuropsychopharmacologicum (CINP), 17(9), 1443-52. doi:10.1017/S1461145714000418

Slaby, I., Holmes, A., Moran, J. M., Eddy, M. D., Mahoney, C. R., Taylor, H. A., \& Brunye, T. T. (2015). Direct current stimulation of the left temporoparietal junction modulates dynamic humor appreciation. Neuroreport, 26, 988-993.

Wirth, M., Rahman, R. A., Kuenecke, J., Koenig, T., Horn, H., Sommer, W., \& Dierks, T. 
(2011). Effects of transcranial direct current stimulation (tDCS) on behaviour and electrophysiology of language production. Neuropsychologia, 49(14), 3989-98. doi:10.1016/j.neuropsychologia.2011.10.015

Yang, L.-Z., Zhang, W., Shi, B., Yang, Z., Wei, Z., Gu, F., .. Rao, H. (2014). Electrical stimulation over bilateral occipito-temporal regions reduces N170 in the right hemisphere and the composite face effect. PloS One, 9, e115772.

Yovel, G., \& Kanwisher, N. (2004). Face perception: domain specific, not process specific. Neuron, 44(5), 889-98. doi:10.1016/j.neuron.2004.11.018 


\section{Figure Legend}

Figure 1. An example face trial, with load level 3, and a foil (new) probe. Note that there was a $200 \mathrm{~ms}$ ISI between each of the 4 encoding stimuli.

Figure 2. Modeled current propagation targeting the right FFA (upper panel) and electrode montage (lower panel).

Figure 3. Mean and standard error Cowan's Kfor face trials as a function of the stimulation condition $(0.5 \mathrm{~mA}, 1.5 \mathrm{~mA})$ and memory load. Control group data plotted for comparison purposes.

Figure 3. Mean and standard error Cowan's $K$ for house trials as a function of the stimulation condition $(0.5 \mathrm{~mA}, 1.5 \mathrm{~mA})$ and memory load. Control group data plotted for comparison purposes.

Figure 5. Scatterplot with linear fit, relating mean Cowan's Krates for faces trails and CFMT scores. Note that both control and experimental groups are depicted. 

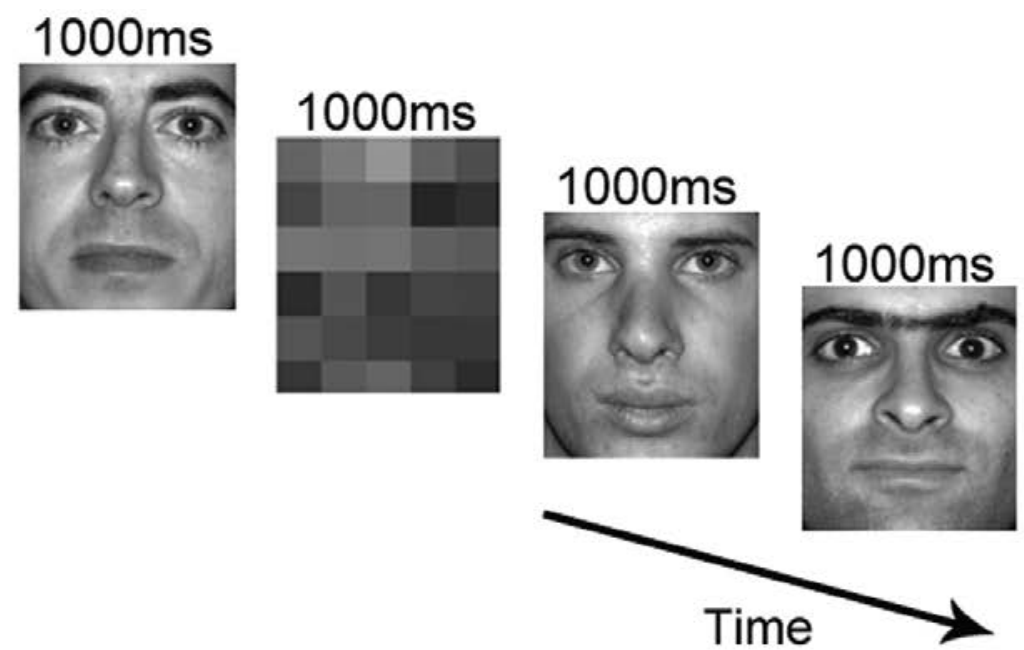

(delay)

4000ms (probe)

4000ms

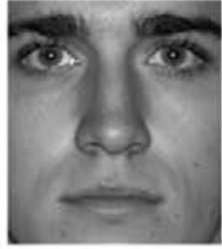

Figure 1 


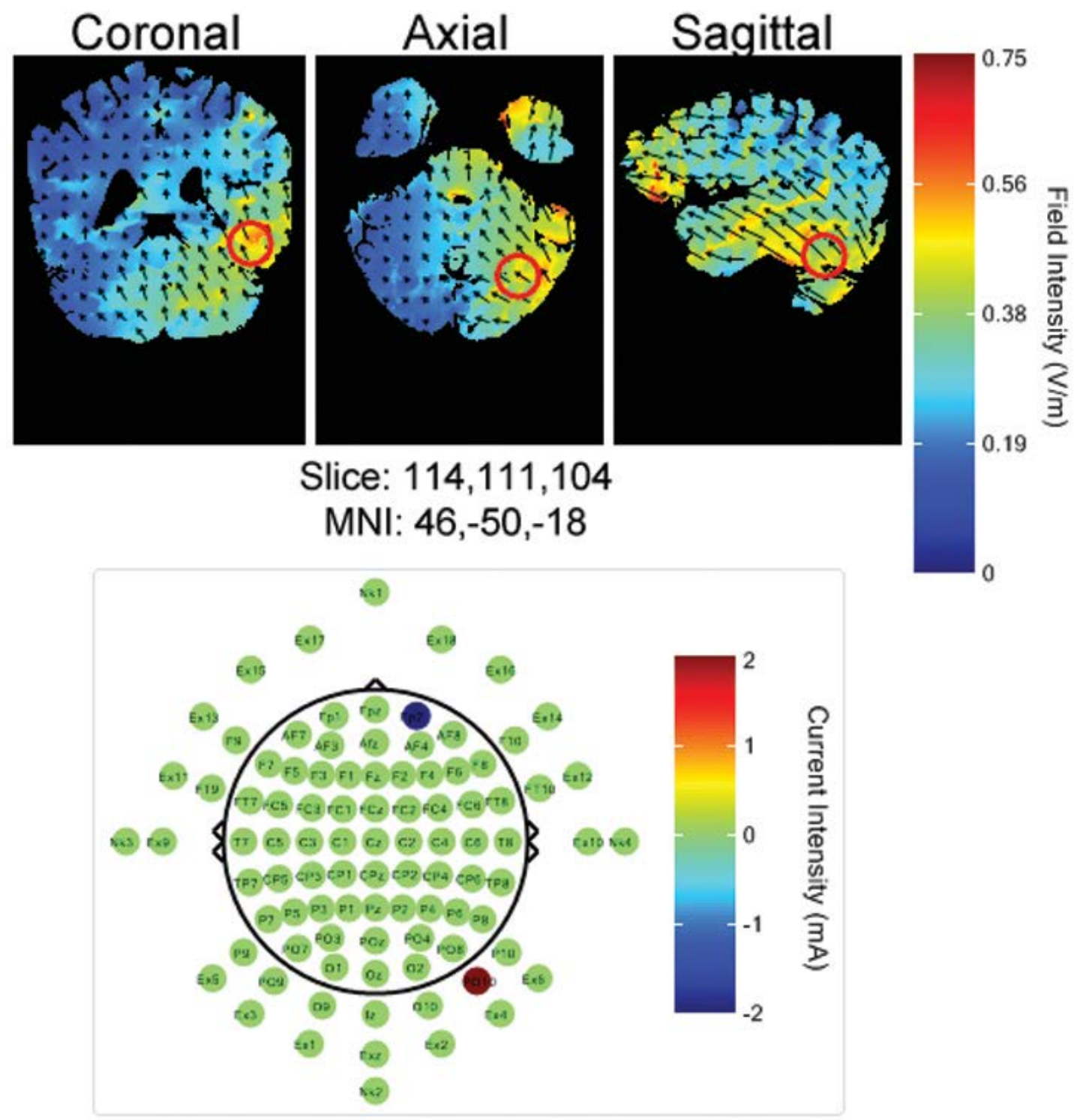

Figure 2 


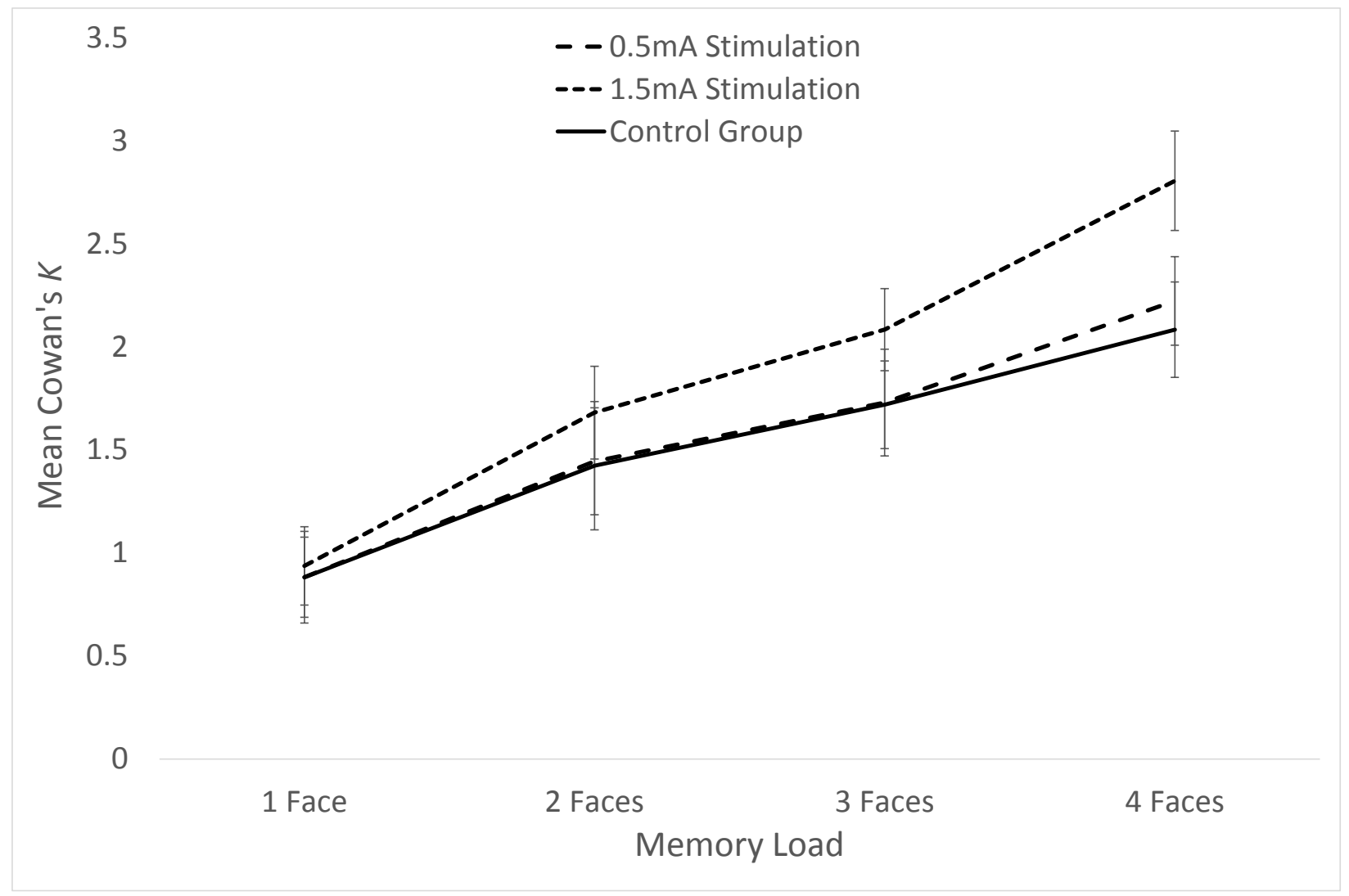

Figure 3 


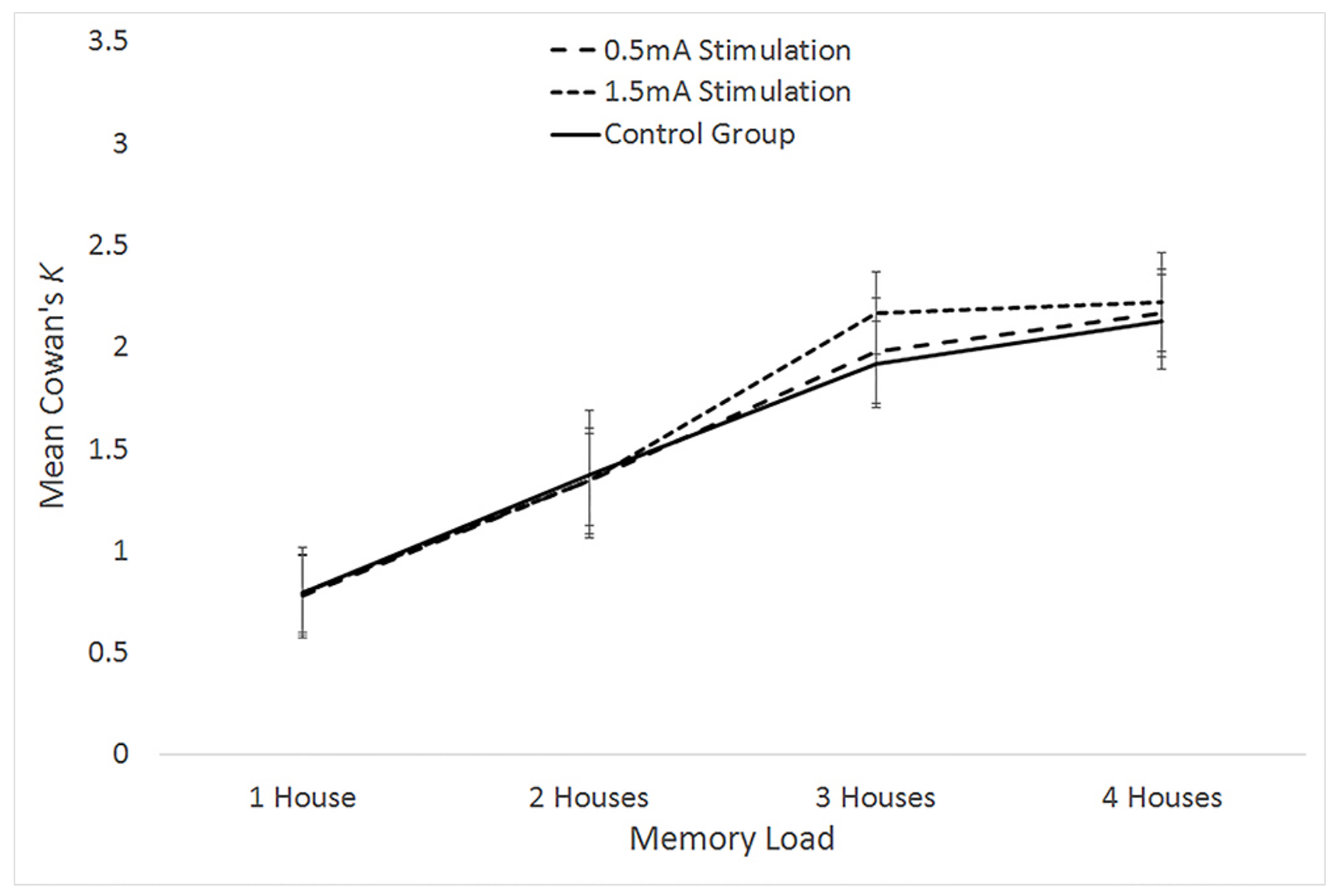

Figure 4 


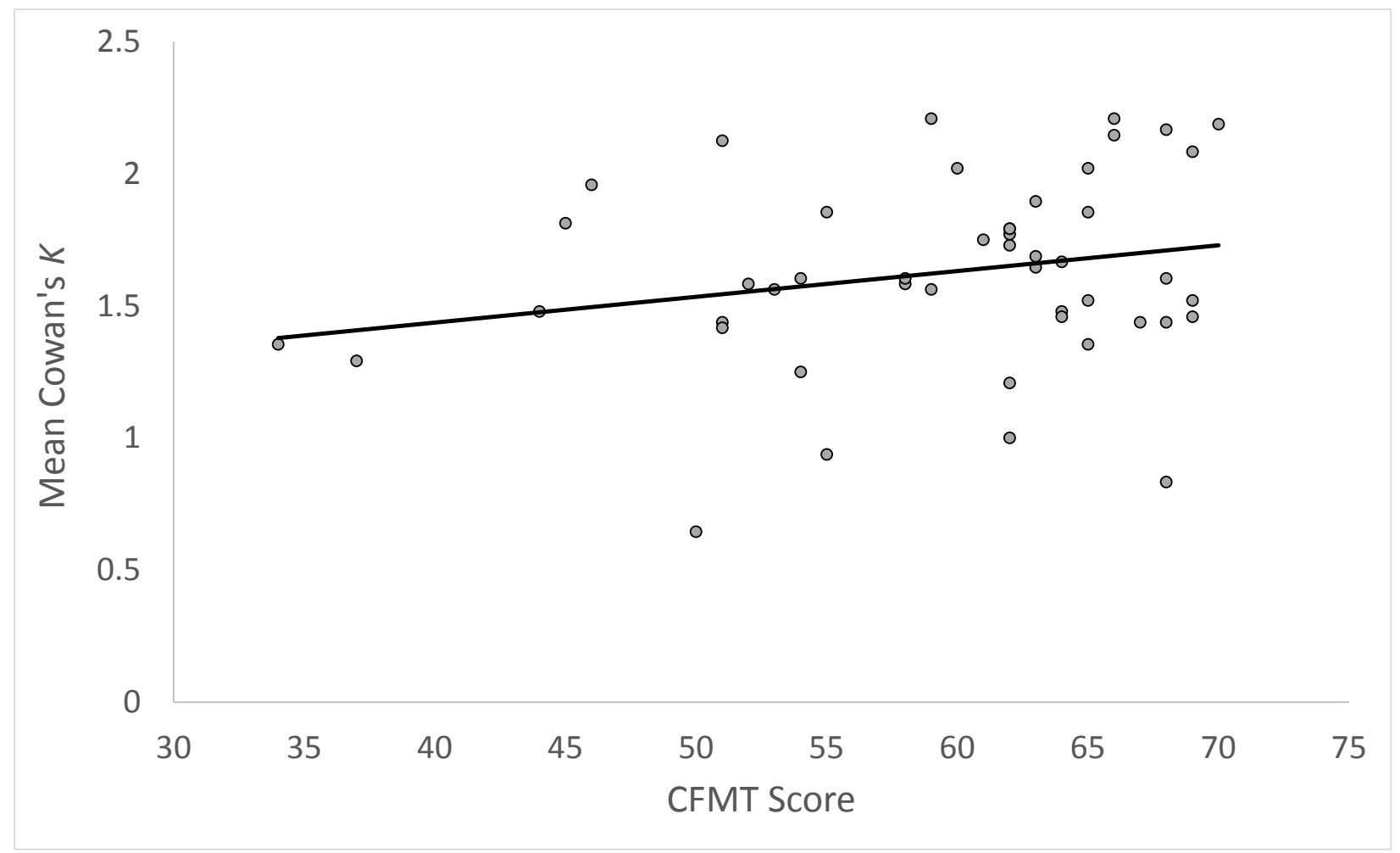

Figure 5 
Table 1. Mean (SD) Cowan's K, hit rates, false alarm rates, $d$-prime, response times, and response criterion, as a function of group, stimulation condition, stimulus category, and memory load.

\begin{tabular}{|c|c|c|c|c|c|c|}
\hline $\begin{array}{c}\text { Dependent } \\
\text { Variable }\end{array}$ & Group & Condition & $\begin{array}{l}\text { Memory } \\
\text { Load } 1\end{array}$ & $\begin{array}{c}\text { Memory } \\
\text { Load } 2\end{array}$ & $\begin{array}{c}\text { Memory } \\
\text { Load } 3\end{array}$ & $\begin{array}{c}\text { Memory } \\
\text { Load } 4\end{array}$ \\
\hline \multirow{6}{*}{ Cowan's $K$} & \multirow{4}{*}{ Experimental } & $0.5 \mathrm{~mA}$ Faces & $.88(.1)$ & $1.44(.4)$ & $1.73(.8)$ & $2.22(.8)$ \\
\hline & & $1.5 \mathrm{~mA}$ Faces & $.94(.1)$ & $1.68(.3)$ & $2.08(.6)$ & $2.81(.8)$ \\
\hline & & $0.5 \mathrm{~mA}$ Houses & $.78(.3)$ & $1.34(.6)$ & $1.98(.8)$ & $2.17(1)$ \\
\hline & & $1.5 \mathrm{~mA}$ Houses & $.79(.2)$ & $1.35(.4)$ & $2.17(.7)$ & $2.22(1)$ \\
\hline & \multirow{2}{*}{ Control } & Faces & $.88(.2)$ & $1.42(.6)$ & $1.72(.6)$ & $2.08(.9)$ \\
\hline & & Houses & $.79(.3)$ & $1.38(.6)$ & $1.92(.9)$ & $2.13(1)$ \\
\hline \multirow{6}{*}{ Hit Rate } & \multirow{4}{*}{ Experimental } & $0.5 \mathrm{~mA}$ Faces & $.93(.1)$ & $.85(.2)$ & $.76(.2)$ & $.74(.2)$ \\
\hline & & $1.5 \mathrm{~mA}$ Faces & $.97(.1)$ & $.93(.1)$ & $.87(.2)$ & $.84(.2)$ \\
\hline & & $0.5 \mathrm{~mA}$ Houses & $.85(.2)$ & $.75(.2)$ & $.78(.2)$ & $.72(.2)$ \\
\hline & & $1.5 \mathrm{~mA}$ Houses & $.86(.2)$ & $.76(.2)$ & $.77(.2)$ & $.69(.2)$ \\
\hline & \multirow{2}{*}{ Control } & Faces & $.92(.2)$ & $.84(.2)$ & $.77(.2)$ & $.72(.2)$ \\
\hline & & Houses & $.89(.2)$ & $.75(.2)$ & $.75(.2)$ & $.69(.2)$ \\
\hline \multirow{6}{*}{$\begin{array}{c}\text { False Alarm } \\
\text { Rate }\end{array}$} & \multirow{4}{*}{ Experimental } & $0.5 \mathrm{~mA}$ Faces & $.05(.1)$ & $.13(.1)$ & $.19(.2)$ & $.19(.2)$ \\
\hline & & $1.5 \mathrm{~mA}$ Faces & $.03(.1)$ & $.09(.1)$ & $.17(.2)$ & $.14(.2)$ \\
\hline & & $0.5 \mathrm{~mA}$ Houses & $.08(.2)$ & $.08(.2)$ & $.12(.2)$ & $.17(.2)$ \\
\hline & & $1.5 \mathrm{~mA}$ Houses & $.07(.1)$ & $.08(.1)$ & $.05(.1)$ & $.17(.2)$ \\
\hline & \multirow{2}{*}{ Control } & Faces & $.03(.1)$ & $.13(.2)$ & $.19(.2)$ & $.19(.2)$ \\
\hline & & Houses & $.11(.2)$ & $.07(.2)$ & $.11(.2)$ & $.16(.2)$ \\
\hline \multirow{3}{*}{ d-prime } & \multirow{3}{*}{ Experimental } & $0.5 \mathrm{~mA}$ Faces & $3.8(.95)$ & $2.8(1.3)$ & $2.1(1.3)$ & $1.9(1.1)$ \\
\hline & & $1.5 \mathrm{~mA}$ Faces & $4.2(.93)$ & $3.5(1.1)$ & $2.7(.98)$ & $2.7(1.2)$ \\
\hline & & $0.5 \mathrm{~mA}$ Houses & $3.4(1.4)$ & $2.8(1.4)$ & $2.8(1.5)$ & $1.9(1.2)$ \\
\hline
\end{tabular}




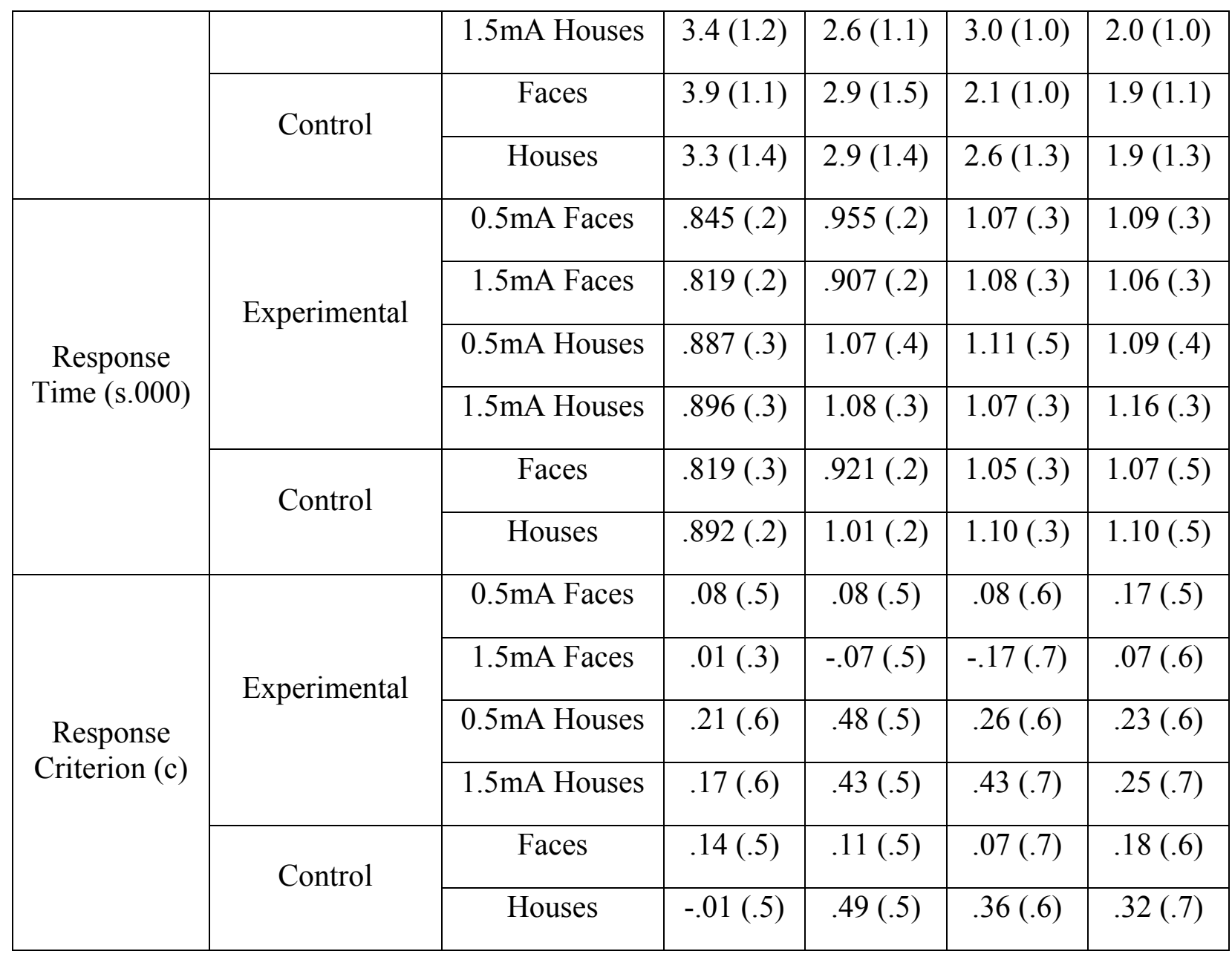

